\title{
A Model-Based Feedback Control Strategy for Heap Bioleaching Processes
}

\author{
Boris I. Godoy, Julio H. Braslavsky and Juan C. Agüero
}

\begin{abstract}
In this paper we propose a feedback control strategy to increase the copper recovery in a heap bioleaching process. The control strategy is implemented using Internal Model Control based on a multivariable linear state-space model obtained by the Maximum Likelihood method. The linear model describes the variations of the average temperature around a nominal trajectory. This trajectory is obtained as the response to nominal inputs that have been proved to work well in real leaching applications, and is computed using a comprehensive high complexity mathematical model developed by BHP Billiton Innovation. The results show that significant increments in copper extraction can be obtained using limited control actions with the proposed feedback strategy.
\end{abstract}

\section{INTRODUCTION}

This paper proposes a simple feedback control strategy to improve mineral extraction in heap bioleaching processes for copper sulphidic ores. Mineral heap leaching is a mining technology based on the dissolution of minerals by a percolating solution through large piles of crushed copper ore. In heap bioleaching the mineral extraction is enhanced by the catalytic action of naturally occurring bacteria. Bioleaching appears as a lower-cost and more environmentally friendly alternative to smelting for the production of high purity cathodic copper from low grade sulphidic ores [1].

Although heap bioleaching process has been used for copper extraction in stand-alone facilities worldwide for many years, its efficiency has been marred by lower than expected production rates and longer than expected start up times [2]. Hence in recent years, the interest of mining companies in developing control and optimisation strategies to improve the technology has increased, leading to greater research efforts to understand the mechanisms that make the process work better [3], [4].

The present work aims to contribute to the optimisation of the bioleaching technology by utilising model-based feedback control methods - to the best of our knowledge, without precedents in the literature. On one hand, typical heap bioleaching facilities around the world operate in essentially an open-loop mode, with fixed set-points for the entire life of the process (sometimes of the order of two years long for leaching of chalcopyrite [5]). On the other hand, although there exist accurate models to describe important

This work was partially supported by BHP-Billition Innovation.

B.I. Godoy is with the School of Electrical Engineering \& Computer Science, The University of Newcastle, NSW 2308, Australia boris.godoydstudentmail. newcastle.edu. au

J.H. Braslavsky and J.C. Aguero are with the Centre for Complex Dynamic Systems \& Control, The University of Newcastle, NSW 2308, Australia julio.braslavsky, juan.aguero@newcastle.edu.au aspects of the process such as oxidation, bacterial growth, and temperature [6], [2], [4], such models are typically of a great mathematical complexity, which makes the design of model-based control strategies very hard.

In our approach we consider an important model estimation aspect well known in the area of identification for control: very often, a relatively simple model suffices as the basis for successful control design for complex processes, as long as essential dynamic aspects of the process are captured by the model [7]. Having this statement in mind, in Section II we focus on the estimation of a simple multivariable statespace linear model maximising the Likelihood function [8].

To generate model estimation data we use a comprehensive high complexity mathematical model developed by BHPBilliton, which we shall refer to as the BHPB model. This proprietary model has of the order of 45 state-space variables and 100 tuning parameters, and operates as a black-box, in that the user is allowed to set up its inputs and has access to its outputs once the model has been run, but has no access to internal computations or structure. The BHPB model has been validated against real data from an experimental bioleaching test column. Given the typical proportions of real heap bioleaching implementations (several square kilometres in area) and time scales (transients of the order of months in the leaching of chalcocite), the use of accurate models such as the BHPB model appear as a necessary step in devising effective feedback control strategies for these processes [9]

A reduced number of input variables for control is selected based on an open-loop sensitivity analysis performed on the BHPB model (Section II-B). Such sensitivity analysis quantifies the effect of small input variations around nominal set-point values on the total amount of extracted copper after a full life-cycle of the heap.

The output of interest to regulate in the present paper is the average temperature in the heap. We use the Expectation Maximisation (EM) algorithm to find linear Maximum Likelihood (ML) model estimates [10], [11]. The linear model obtained describes incremental variations of the output of interest to the selected control inputs around nominal trajectories, which are generated with the BHPB model under nominal conditions. The perturbations on the nominal values of the inputs are generated as multisine signals with an important component of low frequencies [12, chap.4].

In Section III we use the linear incremental model estimated following the procedures described in Section II to design a feedback controller using the Internal Model Control architecture [13], [14], to regulate small increments around the nominal values of the outputs. The closed-loop results 
simulated using the BHPB model are discussed in Section III-B and show around $4 \%$ improvement in the total mass of extracted copper. These results appear very promising given the extreme simplicity of the estimated model (third order) used, and the limited (incremental) action of the control inputs applied, and indicates good potential for feedback operation of heap bioleaching processes.

Note that the proposed approach requires a priori knowledge of the nominal trajectory of interest for model estimation and control design. In an implementation on a real heap, the BHPB model could be used to predict the heap nominal trajectories with periodic parameter tuning to fit its predictions to the real heap measured trajectories. Therefore, further work will concentrate on such implementation structure by considering robustness and measurement noise issues.

\section{Model IDENTIFICATION}

\section{A. The Process}

In copper heap leaching, large heaps of up to several square kilometres by 6 to 10 metres height of crushed copper mine tailings are formed. A sulphuric acid solution, called raffinate, is sprinkled by means of an arrangement of drip lines at the top of the heap. As the solution percolates down through the heap, it becomes enriched by the copper dissolved from the heaped ore, forming the pregnant leach solution (PLS). The PLS is then collected at the base of the heap by an impervious liner and pumped to an electrowinning extraction plant, which produces cathodic copper of $99.99 \%$ purity. The residual solution is then recycled as raffinate to the top of the heap. The process is illustrated in Fig. 1.

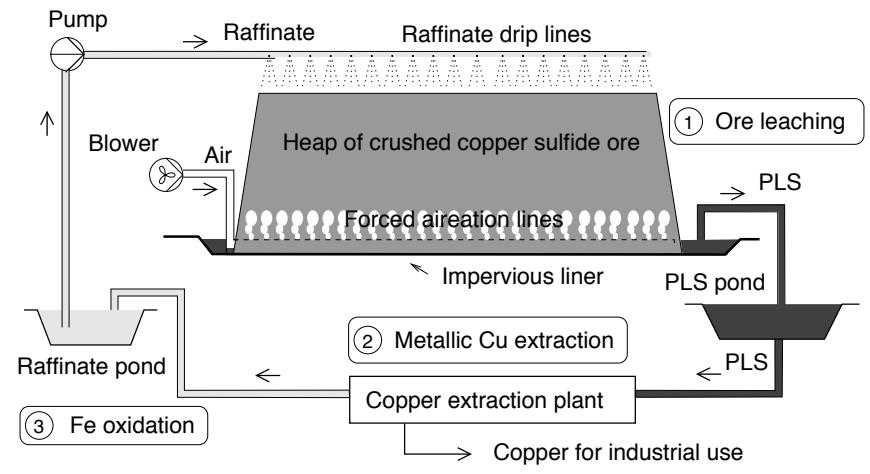

Fig. 1. Simplified copper heap leaching process

To tackle the complexity of this process, we consider a single geometric dimension of the process, namely, the vertical direction, assuming process homogeneity in every direction on the horizontal plane. Given that there are three fundamental inter-coupled sub-processes in bioleaching [15], [4], we focus on the identification of a model for the average temperature, which is one of these three sub-processes which directly affects bacteria population and thereby, efficiency of copper extraction [2].

\section{B. Input Selection by Sensitivity Analysis}

A sensitivity analysis of a selected set of potential control variables is carried out to determine the input variables with best control authority to affect copper extraction.

The candidate manipulated variables are:

- Irrigation rate $\left(F_{i}\right)$ and input Acid Concentration $\left(\mathrm{H}_{2} \mathrm{SO}_{4}\right)$,

- Aeration Rate $\left(A_{i}\right)$ and Raffinate Temperature $\left(T_{i}^{o}\right)$,

- Input Ferric Sulphate Concentration $\left(\mathrm{Fe}_{2}\left(\mathrm{SO}_{4}\right)_{3}\right)$ and input Ferrous Sulphate Concentration $\left(\mathrm{FeS}_{4}\right)$,

- Air Humidity and Air Temperature.

The sensitivity analysis of these variables is carried out by running the BHPB model. We choose each pair of variables listed above and variate them by small values around their nominal set point values to obtain a 2 -dimensional grid. Then the BHPB model is run with the set point values defined by the grid, while keeping all other variables at nominal set point values.

The effect of the set point in the open loop process is quantified using the functional defined by

$$
J=\sum_{k=1}^{N} C_{\mathrm{Cu}}[k] F_{o}[k] e^{-\theta k}, \quad \text { in }\left[g / h . m^{2}\right],
$$

where $C_{\mathrm{Cu}}[k]$ is the concentration of copper in $[g / L]$ and $F_{o}[k]$ is outflux in $\left[L / h \cdot m^{2}\right]$. The functional (1) considers the total copper extracted (per hour $\times m^{2}$ ) during the bioleaching process for a given set-point. Thus, for each pair of manipulated variables, and for each point in the grid of set-point variations, we obtain a value $J$ of total copper extracted. The exponential factor $e^{-\theta k}$ weights more favourably runs of the BHPB model that render copper more rapidly. We choose $\theta=4.8110^{-5}$, which corresponds to a value decrease of approximately $10 \%$ in one year; around 3000 samples when the process is sampled every 4 hrs. This exponential term is very small when time approaches the last samples in the simulation (at about 3000 samples). In this way, runs that extract copper over a long period of time are penalised over runs that extract the same amount of copper in a shorter period of time.

The results obtained are shown in the following plots. Fig. 2 (a) shows sensitivity of $J$ with respect to raffinate influx $\left(F_{i}\right)$ and acid concentration $\left(\mathrm{H}_{2} \mathrm{SO}_{4}\right)$; and Fig. 2 (b) shows the corresponding for aeration rate $\left(A_{i}\right)$ and raffinate temperature $\left(T_{i}\right)$. We observe in Fig. 2 (a) a monotone relationship between input variables and the functional value, which increases when variables do. On the other hand, in Fig. 2 (b), there is an interesting aspect. Namely, there is an optimal extremum value for aeration rate. The existence of such local extremum in copper extraction with respect to aeration rate is reasonable, since we should expect a less productive heap with little or no aeration [16], but also with excessive aeration, since then the heap will be cooled down.

The effect of the pair Ferric Sulphate $\left(\mathrm{Fe}_{2}\left(\mathrm{SO}_{4}\right)_{3}\right)$ and Ferrous Sulphate $\left(\mathrm{FeSO}_{4}\right)$ is shown in Fig. 3 (a). We can observe from Fig. 3 (a) that increasing the values for each of the variables increases the copper extraction, but its effect is 
less notorious than, for example, the pair Aeration Rate and Raffinate Temperature.

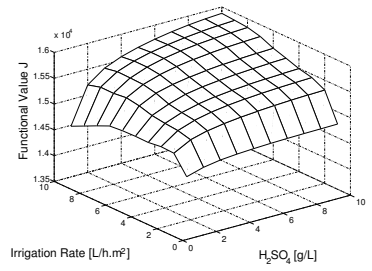

(a) Functional Value varying $F_{i}$ and $\mathrm{H}_{2} \mathrm{SO}_{4}$.

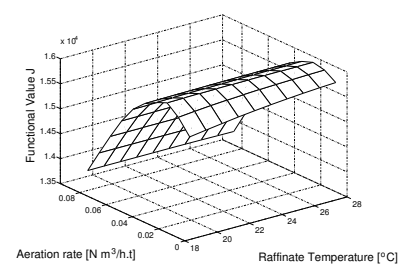

(b) Functional Value varying Aeration Rate and Raffinate
Temperature.
Fig. 2. Functional Value varying different possible manipulated variables.

Finally, the last pair considered is given by Air Humidity and Air Temperature. As we can see in Fig. 3 (b), they have little effect on copper extraction. Thus, we discard them as possible control variables.
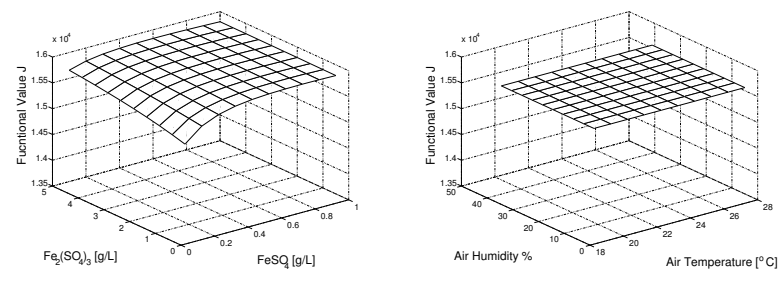

(a) Functional Value varying $\mathrm{Fe}_{2}\left(\mathrm{SO}_{4}\right)_{3}$ and $\mathrm{FeSO}_{4}$.

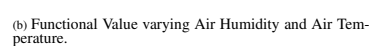

Fig. 3. Functional Value varying different possible manipulated variables.

We conclude from this sensitivity analysis that the most influential inputs affecting the bioleaching process in open loop are: Influx $\left(F_{i}\right)$, input sulphuric acid concentration $\left(\mathrm{H}_{2} \mathrm{SO}_{4_{i}}\right)$, input raffinate temperature $\left(T_{i}^{o}\right)$ and aeration rate $\left(A_{i}\right)$. We expect that these variables also affect significantly the process in closed loop.

\section{Input signal selection}

Once we have identified the most influential variables and in order to drive the process to a better copper recovery performance, we need to design inputs to the system in order to collect the data which will be used in the routines for identification.

Notice that in this paper, the improvement on copper extraction will be done indirectly regulating the average temperature. We discard the raffinate temperature as an input variable since it is obvious that the average temperature in the heap raises if raffinate temperature does. We want to explore with the remaining variables which seem to be easier to implement. Within these variables, we also discard the sulphuric acid concentration because it has little effect on the temperature [17].

In the average temperature modelling, the selected input variables $F_{i}$ and $A_{i}$ persistently excite the BHPB model as shown in Fig. 4.

The multisine signals generated as inputs change about $\pm 70 \%$ around the nominal values (dashed line). These nominal values have been proven to be efficient in copper recovery

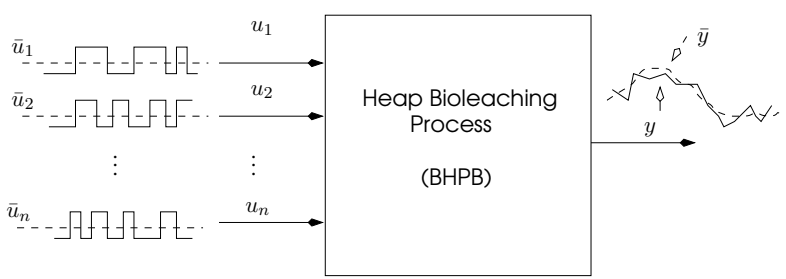

Fig. 4. Exciting the BHPB model with signals (solid line) around nominal values (dashed line).

in an open loop scheme. As output, we obtain a signal varying around the nominal output (dashed line) as seen in Fig. 4.

We generate two sequences of inputs as shown in Fig. 5. The first sequence (thick line) is used for estimation and the second one (thin line) is used for a posteriori validation of the obtained model. Notice that the data, in Fig. 5, shows
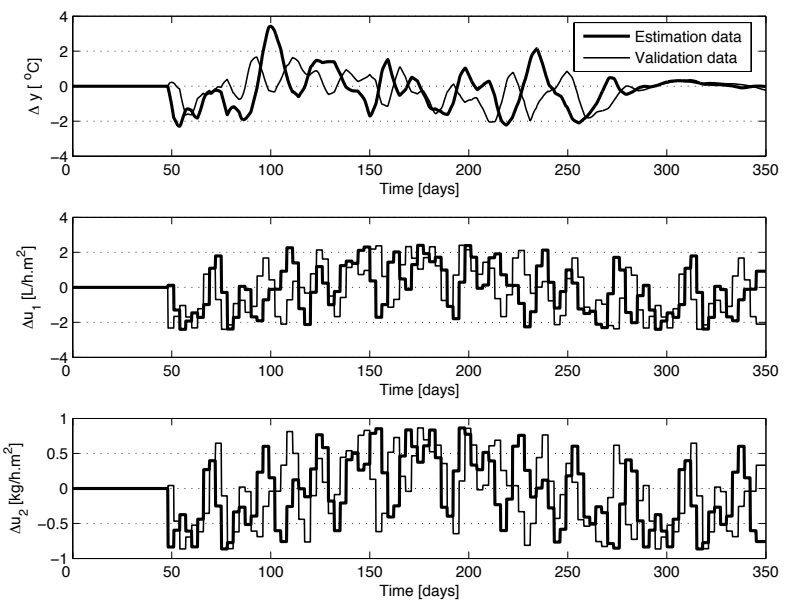

Fig. 5. Data for the modelling of the average temperature. Excited selected inputs and outputs for estimation (thick line) and validation (thin line).

the variation of temperature with respect to its nominal trajectory, and it considers only the values after day 50th because the BHPB model shows little effect on the outputs before that day, to any kind of variation in the inputs [17]. Also, notice that in the same figure, after approximately 300 [days], the BHPB model does not have a significant response to the selected inputs. This is expected as the temperature, in a real heap bioleaching process, will start stabilising around a low set-point after most of the chemical reactions are exhausted. This physical phenomena has an impact on the data to be consider in the routines for identification. In fact, we will only consider the significant data, for estimation and validation, in the range of $[50,300]$ days.

In a general case, with noise acting on the system, the model to be found is in the following form:

$$
\begin{aligned}
& x_{t+1}=A_{n \times n} x_{t}+B_{n \times n_{u}} u_{t}+w_{t} \\
& y_{t}=C_{n_{y} \times n} x_{t}+D_{n_{y} \times n_{u}} u_{t}+v_{t},
\end{aligned}
$$

where $x_{t} \in \mathbb{R}^{n}, y_{t} \in \mathbb{R}^{n_{y}}$, and $u_{t} \in \mathbb{R}^{n_{u}}$ are the state, the output and the input of the system, respectively. The 
vector $\eta_{t}=\left[\begin{array}{ll}w_{t} & v_{t}\end{array}\right]^{T}$, which combines $w_{t}$ and $v_{t}$, describes the process and output noise respectively. The disturbance input $\eta_{t}$ is assumed to be a zero mean Gaussian process with covariance matrix given by

$$
\mathrm{E}\left\{\eta_{t} \eta_{t}^{T}\right\}=\left[\begin{array}{cc}
Q & 0 \\
0 & R
\end{array}\right] .
$$

The initial state $x_{0}$ is assumed to be Gaussian with mean $\mu$ and covariance $P_{0}: x_{0} \sim \mathrm{N}\left(\mu, P_{0}\right)$.

In this case, to the data generated by the BHPB model and shown in Fig. 5, has been added a noisy term to the output to account for the uncertainty of the measurements. Notice that, the noisy terms given in (2) also account for the model uncertainty as we are modelling a nonlinear system using a linear approximation.

The procedure for obtaining the matrices $A, B, C$ and $D$ in (2) is explained in the following Section.

\section{Review of the Expectation Maximisation algorithm}

In order to obtain a model for the average temperature in the heap bioleaching process, we use an iterative algorithm called Expectation Maximisation (EM). EM provides the Maximum-Likelihood (ML) estimate for a vector of parameters $\theta_{o}$. We choose a ML approach because of the desirable properties of the estimators such as: consistency, unbiasedness and efficiency [18], [8]. In the ML framework, the following log-likelihood function is maximised:

$$
l(\theta)=\log p\left(Y_{1: N} \mid \theta\right),
$$

where $Y_{N}$ denotes the given data set containing the system outputs i.e. $Y_{1: N}:=\left\{y_{1}, y_{2}, \ldots, y_{N}\right\}$. For future use, we also introduce the state sequence $X_{1: N}:=\left\{x_{1}, \ldots, x_{N}\right\}$.

The EM algorithm may be summarised as follows [10], [19]:

1) Choose an initial estimate $\hat{\theta}_{0} \in \Omega$, where $\Omega$ is a constraint set in the parameter space.

Then, for $i=0,1, \cdots$

2) E-step: Compute the auxiliary function $\mathbf{Q}\left(\theta, \hat{\theta}_{i}\right)$ which is the expected value of the complete data loglikelihood with respect to the random variable $X_{1: N}$ (usually called "hidden data" in the statistics literature) given the observed data $Y_{1: N}$ and the previous estimate $\hat{\theta}_{i}$ :

$$
\mathbf{Q}\left(\theta, \hat{\theta}_{i}\right)=\underset{X_{1: N}}{\mathrm{E}}\left\{\log \left[p\left(X_{1: N}, Y_{1: N} \mid \theta\right)\right] \mid Y_{1: N}, \hat{\theta}_{i}\right\}
$$

3) M-step: Set $\hat{\theta}_{i+1}=\underset{\theta \in \Omega}{\arg \max } \mathbf{Q}\left(\theta, \hat{\theta}_{i}\right)$.

4) Go to step 2, and continue until convergence.

Steps 2 and 3 are usually known as the E-Step and M-Step respectively. Under quite general conditions [10], [20], [21], the EM algorithm can be proven to converge to a stationary point of the likelihood function which in many practical applications will be a local maximum of the likelihood function [22].

The advantage of using the EM (apart from the usual statistical properties of a ML estimator) to identify a linear state-space model is that the algorithm is simple and has a closed form solution for both steps.

\section{E. EM applied to linear state space models}

To apply the EM algorithm for the problem of interest in the current paper we assume that the data has been generated by the model given in (2).

The parameters to be identified are $\theta=$ $\left(\vec{A}^{T}, \vec{B}^{T}, \vec{C}^{T}, \vec{D}^{T}, \mu^{T}, \vec{P}_{0}^{T}, \vec{Q}, \vec{R}\right)^{T} \quad$ where the arrow $(\overrightarrow{.})$ denotes the vec operator which creates a vector from a matrix by stacking its columns [23]. The state vector, $x_{k}$, as the hidden variable.

We describe the proposed algorithm under the two headings of $E$ and $M$ step.

The E-step: The E-step requires that we calculate the following

$$
\begin{aligned}
& -2 \mathbf{Q}\left(\theta, \theta_{i}\right)=\left(n+N n_{y}+N n\right) \log 2 \pi+\log \left|P_{0}\right| \\
& +N \log |Q|+N \log |R| \\
& +\operatorname{tr}\left\{P_{0}^{-1}\left[\Sigma_{0 \mid N}+\left(\hat{x}_{0 \mid N}-\mu\right)\left(\hat{x}_{0 \mid N}-\mu\right)^{T}\right]\right\} \\
& +\operatorname{tr}\left\{Q^{-1}\left[[A, B] \Gamma[A, B]^{T}-\Psi[A, B]^{T}-[A, B] \Psi^{T}+\Phi\right]\right\} \\
& +\operatorname{tr}\left\{R^{-1}\left[[C, D] \Pi[C, D]^{T}-\Lambda[C, D]^{T}-[C, D] \Lambda^{T}+\Delta\right]\right\},
\end{aligned}
$$

where $|A|=\operatorname{det}(A), \operatorname{tr}(A)=\sum a_{i i}$, and where the matrices $\Delta, \Phi, \Pi, \Gamma, \Lambda$ and $\Psi$ can be calculated as shown in [17].

The M-step: In the M-step, the estimates have the following closed form (just set the derivatives of $\mathbf{Q}\left(\theta, \theta_{i}\right)$ equal to zero and solve for $\theta$ ):

$$
\begin{aligned}
{[A, B] } & =\Psi \Gamma^{-1} \\
{[C, D] } & =\Lambda \Pi^{-1} \\
Q & =\frac{1}{N}\left[\Phi-\Psi \Gamma^{-1} \Psi^{T}\right] \\
R & =\frac{1}{N}\left[\Delta-\Lambda \Pi^{-1} \Lambda^{T}\right] \\
\mu & =\hat{x}_{0 \mid N} \\
P_{0} & =\Sigma_{0 \mid N} .
\end{aligned}
$$

\section{F. Model Validation}

Once the model has been obtained, it is necessary to decide if the model is "good" enough for our purposes. The required degree of accuracy of the obtained model will ultimately depend on the final application [8, pg.509]. For example, if the application is the regulation of one chosen output of the system, then the accuracy of the model may be less demanding than if the model is intended to predict or to get new insights about the system.

In our example, we apply the input validation data in Fig. 5 , to both the linear obtained model with state-space matrices $A, B, C, D$ in (14) and to the BHPB model. The results of this test are presented in [17], and not included here for simplicity purposes.

\section{Control Design}

We now discuss one feedback configuration to improve the copper extraction in an aerated heap. The configuration takes the average temperature in the heap as the control objective. 
We initially focus on the temperature as control objective because temperature is related to the bacterial activity [16]. We can expect that maintaining high temperature for longer periods of time will improve bacterial activity and consequently make the copper extraction more efficient.

We implement the IMC strategy considering control of the average temperature in the heap.

\section{A. Brief Review of IMC}

A typical Internal Model Control scheme [14], [13] is shown in Figure 6. The following relationships can be established

$$
\begin{aligned}
\Delta u & =[I+G(P-\tilde{P})]^{-1} G(\Delta r-d) \\
\Delta y & =P[I+G(P-\tilde{P})]^{-1} G(\Delta r-d)+d,
\end{aligned}
$$

where $I$ is the identity matrix, $P$ is the plant, $\tilde{P}$ is a model of the plant, and $G$ is a controller. Signals $\Delta r, \Delta y, d, \Delta u$ are defined as reference, output, disturbance and control action respectively. The output of the model is defined as $\Delta y_{m}$.

In this case, $\Delta$ denotes the variation of a variable with respect to its nominal value, e.g., $\Delta u=u-\bar{u}$ and $\Delta y=$ $y-\bar{y}$, where $\bar{u}$ and $\bar{y}$ are the nominal values for the inputs and outputs, respectively.

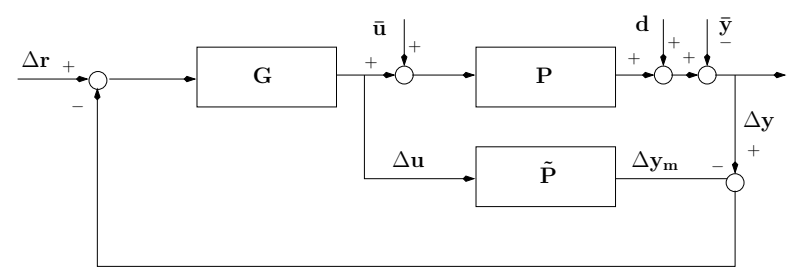

Fig. 6. Classical IMC configuration.

In IMC we intend to use as $G$ the inverse of the model for the plant, multiplied by a function $F_{d}$ used to adjust the bandwidth of the closed loop and to account for uncertainties in the model [14]. Due to the non-square characteristic of the model $\tilde{P}$ (the model of the plant has different number of inputs and outputs), we use a generalised inverse type 1 of $\tilde{P}$ as defined in [25]. In particular we choose

$$
G=F_{d} \tilde{P}^{T}\left[\tilde{P} \tilde{P}^{T}\right]^{-1},
$$

where the filter $F_{d}$ is a scalar bi-proper transfer function. Notice that the inverse of the term $\tilde{P} \tilde{P}^{T}$ is, in general, well defined since it is a scalar transfer function. In the case when non-minimum phase zeros are present, we replace them by using their corresponding mirrored ones (with respect to the unit circle).

\section{B. Control of average temperature in the heap}

For the control of the average temperature in the heap, we have chosen a third order model. Using the procedure structure explained in Section II-D, we estimate the incremental model for the variations in the average temperature, with matrices A,B,C,D given by

$$
\begin{gathered}
A=\left[\begin{array}{ccc}
0.9188 & -0.0029 & 0.0214 \\
0.2886 & 1.0030 & 0.2311 \\
1.023 & 0.0239 & 0.2161
\end{array}\right], B=\left[\begin{array}{cc}
0.0108 & -0.0627 \\
-0.0344 & -0.5897 \\
-0.5036 & 2.6640
\end{array}\right] \\
C=-\left[\begin{array}{lll}
0.6149 & 0.1054 & 0.0330
\end{array}\right], D=-\left[\begin{array}{ll}
0.0709 & 0.0217
\end{array}\right]
\end{gathered}
$$

The matrices obtained above corresponds to a linear discrete-time model, with sampling time $T=4[h]$.

The results in Fig. 7 show that the linear controller used for the incremental gives consistent results when different $\Delta r$ are applied. These $\Delta r$ corresponds to the desired increment in the output being controlled. In this case, the output being controlled is the temperature, then $\Delta r$ corresponds to the variations for this variable in $\left[{ }^{\circ} \mathrm{C}\right]$. The case for $\Delta r=0$ is the open loop case using nominal inputs.
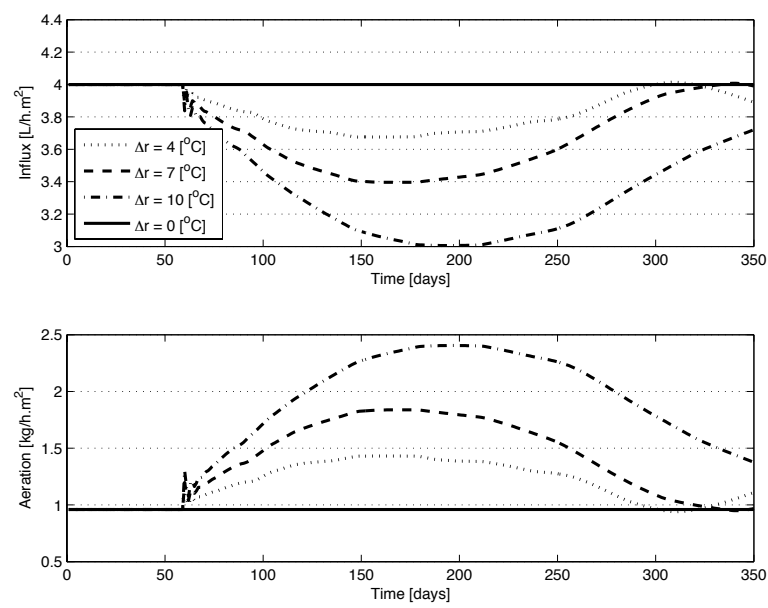

Fig. 7. Response of the average temperature in the BHPB model to different values of $\Delta r$.
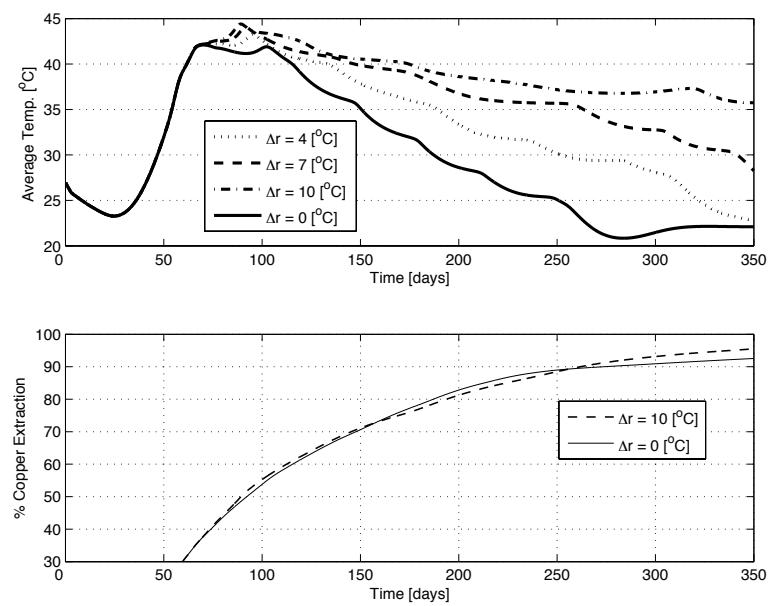

Fig. 8. Control effort in the chosen inputs and \% of copper extraction, regulating the average temperature.

We also notice in Fig. 8 that the control efforts are reasonable in terms of variations regarding the nominal 
values (continuous line). Notice that the control actions are applied once those actions can make a difference in the output of the closed loop, that is, after the day 50th in the process. Before that day, changes in the selected inputs do not produce a visible change in the outputs of interest, then we have designed the control scheme to operate only after the day 50th. The best improvement on copper extraction controlling the temperature is approximately $4 \%$ (seen almost at the end of the heap's life) when the maximum control effort is used (for $\Delta r=10\left[{ }^{\circ} \mathrm{C}\right]$ ).

\section{CONCLUSIONS AND FUTURE WORK}

We have identified a model for the increments in the average temperature. The model has been obtained solving the maximum likelihood (ML) estimators using EM algorithms in a time domain framework.

The use of incremental models for this kind of process on an IMC strategy indicates significant potential improvements on the copper extraction which is our final objective. This improvement on the copper extraction has been carried out controlling the average temperature in the heap.

The drawback of the proposed approach is the requirement of the nominal trajectory for the variable to be controlled. This trajectory is necessary because the models to be estimated are incremental, that means, they describe the variations around a nominal trajectory of the chosen variable. This nominal trajectory is obtained using the BHPB model, but this one is another model and its predicted value could be different to the real one. Hence, as a future research topic, we will focus on a robustness analysis considering that the predicted nominal trajectory by the BHPB model could be slightly different to the real one. This can be done by adding noise to the nominal trajectory and running Montecarlo simulations.

\section{ACKNOWLEDGMENTS}

The authors gratefully acknowledge the contribution of John Truelove from BHP-Billiton Innovation, Newcastle, Australia, for allowing us to use the BHPB model and the data generated by it. We also gratefully acknowledge David Allingham for his invaluable support during the online implementation of the BHPB model.

\section{REFERENCES}

[1] J. Barret, M. Hughes, G. Karavaiko, and P. Spencer, Metal Extraction by Bacterial Oxidation of Minerals. Ellis Horwood Limited, 1993.

[2] D. G. Dixon, "Analysis of heat conservation during copper sulphide heap leaching," Journal of Hydrometallurgy, vol. 58, pp. 27-41, 2000.

[3] M. Leahy, M. Davidson, and M. P. Schwarz, "A two-dimensional cfd model for heap bioleaching of chalcocite," ANZIAM, vol. 46, pp. C439-C457, 2005.

[4] J. Peterson and D. Dixon, "A modelling study of the dynamics of sulphide heap leach processes with a view to improved and novel applications," in 23th International Mineral Processing Congress, Cape Town, South Africa, October 2003.

[5] R. W. Barlett, Solution Mining : Leaching and Fluid Recovery of Materials, 2nd ed. Gordon and Breach Science Publishers, 1998.

[6] G. Pantelis and A. Ritchie, "Rate-limiting factors in dump leaching of pyritic ores," Applied Mathematical Modelling, vol. 15, pp. 553-560, 1992.
[7] M. Gevers, Essays in control: perspectives in the theory and its applications. Birkhauser, 1993, ch. Towards a joint design of identification and control?, pp. 111-151.

[8] L. Ljung, System Identification: Theory for the User, 2nd ed. Upper Saddle River, NJ: Prentice Hall International, Inc., 1999.

[9] J. Petersen and D. G. Dixon, Microbial Processing of Metal Sulfides. Springer-Verlag, 2007, ch. 8: Principles, Mechanisms and Dynamics of Chalcocite Heap Bioleaching.

[10] A. P. Dempster, N. M. Laird, and D. B. Rubin, "Maximum likelihood from incomplete data via the EM algorithm," Journal of the Statistical Society, Series B, vol. 39, no. 1, pp. 1-38, 1977.

[11] R. Shumway and D. Stoffer, "An approach to time serie smoothing abd forecasting using EM algorithms," Division of Statistics, University of Californnia, Davis, Tech. Rep., 1981.

[12] R. Pintelon and J. Schoukens, System Identification: A frequency domain approach. IEEE Press, 2001.

[13] G. Goodwin, S. Graebe, and M. Salgado, Control System Design. Prentice Hall International, Inc., 2001.

[14] C. E. Garcia and M. Morari, "Internal model control. 1. a unifying review and some new results," Ind. Eng. Chem. Process Des. Dev., vol. 21, pp. 308-323, 1982.

[15] B. Godoy, J. Braslavsky, and K. Mahata, "Low complexity modelling in bioleaching processes," in 14th IFAC Symposium on System Identification (SYSID), Newcastle, Australia, 2006.

[16] H. M. Lizama, "Copper bioleaching behaviour in an aerated heap," International Journal of Mineral Processing, vol. 14, 2004.

[17] B. I. Godoy, J. H. Braslavsky, and J. C. Agüero, "A model-based feedback control strategy for heap bioleaching processes," School of EE\&CS, The University of Newcastle, Tech. Rep. EE07010, 2007, ftp : //warhol.newcastle.edu.au/pub/Reports/EE07010.pdf.

[18] G. C. Goodwin and R. Payne, Dynamic System Identification. Academic Press, 1977.

[19] J. C. Agüero, J. I. Yuz, and G. C. Goodwin, "Frequency domain identification of MIMO state space models using the EM algorithm," in European Control Conference ECC, 2007.

[20] C. F. J. Wu, "On the convergence properties of the EM algorithm," The Annals of Statistics, vol. 11, no. 1, pp. 95-103, 1983.

[21] R. A. Boyles, "On the convergence of the EM algorithms," Journal of the Royal Statistical Society, vol. 45, no. 1, pp. 47-50, 1983.

[22] G. J. MacLachlan and T. Krishnan, The EM Algorithm and Extensions. Wiley, 1997.

[23] J. R. Schott, Matrix Analysis for Statistics. New York: John Wiley and Sons, Inc., 1997.

[24] C. Economou, M. Morari, and B. Palsson, "Internal model control. extension to nonlinear systems," Ind. Eng. Chem. Process Des. Dev., vol. 25, pp. 403-411, 1986.

[25] A. Ben-Israel and T. N. E. Greville, Generalized Inverses: Theory and Applications, 2nd ed. Springer-Verlag, 2003. 